\title{
Eine viel zu selten erkannte Störung
}

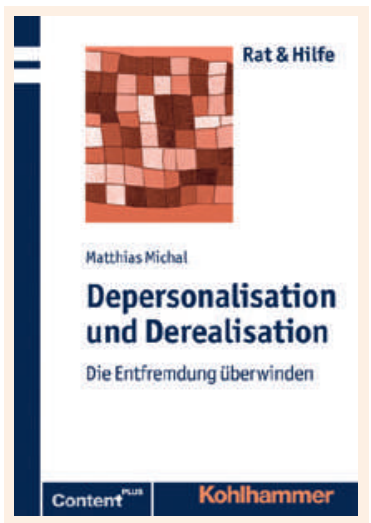

Matthias Michal Depersonalisation und Derealisation

Die Entfremdung überwinden Stuttgart: Kohlhammer Verlag; 2012

120 Seiten. $35.50 \mathrm{CHF}$

ISBN 978-3-17-022170-3
Der Autor stützt sich auf seine mehr als 10-jährige klinische und wissenschaftliche Arbeit mit Menschen, die unter einem Depersonalisations-/Derealisationssyndrom leiden. Er leitet die bis heute einzige Spezialsprechstunde in Deutschland an der Universität Mainz, die sich explizit dieser Störung widmet, die bislang viel zu selten erkannt wird. Dabei ist das Depersonalisations-/Derealisationssyndrom mit einer Punktprävalenz von $0,8-1 \%$ im westlichen Kulturkreis etwa gleich häufig wie Schizophrenien, Epilepsien, Zwangsstörungen oder Magersucht und somit keineswegs eine seltene psychische Erkrankung. Dass diese Störung häufig unerkannt bleibt, ist nur teilweise darauf zurückzuführen, dass Betroffene ihr verändertes, entfremdetes Erleben ihres eigenen Selbst (Depersonalisation) und ihrer Umgebung (Derealisation) oftmals nicht genauer beschreiben können und so häufig nach Metaphern greifen oder in ihren Schilderungen vielfach Begriffe wie «als ob» oder «wie wenn» verwenden. Michal appelliert in seinem Buch vielmehr immer wieder an die Fachleute, die von Betroffenen aufgesucht werden und die Störung nicht erkennen, weil sie dieses Krankheitsbild nicht kennen und die geschilderten Symptome nicht selten einer psychotischen Störung zuordnen. Es resultiert somit eine doppelte Isolation: Einerseits erleben sich Betroffene von sich und ihrer Umwelt abgelöst, und andererseits begegnet ihnen Unverständnis bei denjenigen, von denen sie sich Hilfe und Verständnis erhoffen. Nicht selten resultieren daraus falsche Behandlungsansätze.

Michal gibt zunächst einen umfassenden Überblick über die klinischen Merkmale dieser Störung und die häufigsten komorbiden psychiatrischen Störungen. Im anschliessenden Teil befasst er sich mit den Auslösern und Ursachen dieser Störung, wobei er neben soziokulturellen und psychologischen Faktoren unter Einbezug bindungstheoretischer Aspekte auch neurobiologische Mechanismen bespricht. Situationen, die erhebliche Ängste bis hin zu Panikattacken auslösen - mitunter auch Cannabiskonsum -, führen bei Patienten mit Depersonalisations-/ Derealisationssyndrom zu einer verstärkten Aktivierung des ventralen präfrontalen Kortex, der für die Kontrolle von Emotionen bedeutsam ist, und entsprechend zu einer verminderten Aktivierung sogenannter limbischer Areale, insbesondere der Insula und der Amygdala, womit die klassischen Entfremdungserlebnisse erklärt werden.

Die weiteren Kapitel sind der Behandlung gewidmet. Hier macht Michal keinen Hehl daraus, dass medikamentöse Behandlungen nur in Einzelfällen wirksam sein können. Vielmehr betont er die Wichtigkeit einer kontinuierlichen psychotherapeutischen Begleitung und legt besonderes Gewicht auf die Achtsamkeits- und Meditationspraxis, die dem Bewusstseinszustand der Depersonalisation genau entgegengesetzt ist. Das Depersonalisations-/Derealisationssyndrom kennt verschiedene Verlaufsformen; gemeinsam ist diesen aber, dass die Betroffenen einem erheblichen subjektiven Leiden ausgesetzt sind.

Das Buch ist ein ausgezeichneter Beitrag zu einem Krankheitsbild, das zu den wenig bekannten, aber keineswegs seltenen psychischen Krankheitsbildern gehört. Es ist sowohl Lehrbuch für Fachleute als auch Ratgeber für Betroffene, liest sich leicht und legt in klarer und einprägsamer Sprache die relevanten medizinischen Aspekte dar, und ist daher gleichermassen für Fachleute, Betroffene wie auch für interessierte Laien sehr zu empfehlen.

PD Dr. med. Andor Simon, Basel, Bruderholz 